\title{
Point of Care Blood Ketone and Glucose Concentrations in Full Term Healthy Neonates with in First 48 Hours of Life: The POCK Study
}

\author{
Shradha Joshi, Asavari Kanade and Uday P Devaskar* \\ Deenanath Mangeshkar Hospital, Pune, Maharashtra, India \\ *Corresponding author: Uday P Devaskar, Professor of Pediatrics at David Geffen School of Medicine at UCLA, Los \\ Angeles, California
}

\section{ARTICLE INFO}

Received: 幽 June 05, 2021

Published: 慧 June 16, 2021

Citation: Shradha Joshi, Asavari Kanade, Uday P Devaskar. Point of Care Blood Ketone and Glucose Concentrations in Full Term Healthy Neonates within First 48 Hours of Life: The POCK Study. Biomed J Sci \& Tech Res 36(4)-2021. BJSTR. MS.ID.005871.

Keywords: Ketone bodies; Newborn; Glucose; Point of Care

\begin{abstract}
In a full-term newborn baby, soon after birth, during the metabolic adjustment, there is a brisk response called suckling ketogenesis. These ketone bodies (KB), namely 3 - $\beta$-hydroxyl-butyrate and acetoacetate provide alternative energy to glucose for the CNS. However, quantitation of blood KB is not easy and needs large amount of blood. We studied the ontogeny of blood KB and glucose concentration during the first 48 hours of life in healthy newborn babies $(n=63)$ by using a point of care device (Precision Xtra, Abbott) which requires only a drop of blood. We also examined the blood KB levels in SGA, LGA, IUGR, hypoglycemic and infants born to mothers with diabetes.
\end{abstract}

Abbreviations: AGA: Appropriate for Gestational Age; BW: Birth Weight; FFA: Free Fatty Acids; 3- $\beta$-OHB: 3- $\beta$-Hydroxyl-Butyrate; IDM: Infant of Diabetic Mother; IUGR: IntraUterine Growth Restriction; KB: Ketone Bodies; LGA: Large for Gestational Age; POC: Point of Care; POCK: Point of Care Ketone; SGA: Small of Gestational Age

\section{Introduction}

Metabolic adjustment is the process whereby the newborn adapts from a continuous supply of glucose through the placenta to a fast feed cycle and a diet mostly of maternal milk [1]. In the few hours after birth blood glucose generally declines and is self-limiting even in the infant who is not fed [1]. After the initial decline in blood glucose there is a brisk ketogenic response. This phenomenon, known as suckling ketogenesis, is observed in many mammalian species including the human [1]. These ketone bodies (KB), namely 3 - $\beta$-hydroxyl-butyrate $(3-\beta-\mathrm{OHB})$ and acetoacetate (Ac Ac), produced by the liver from fatty acids, provide alternative energy for the CNS $[1,2]$.

During the first few hours after birth, neonates have low serum $\mathrm{KB}$ concentrations despite adequate levels of free fatty acids (FFA) [2]. Thereafter, healthy term infants show high KB turnover rates.
By 2-3 days they exhibit high KB concentrations [2]. Thus, vigorous ketogenesis is an integral part of the extra-uterine adaptation in a full-term neonate [2]. However, quantitation of blood KB concentration is not generally performed because the regular laboratory method is time consuming, cumbersome needing large amount of blood. Even in quaternary centers like ours, it takes > 4 hours to obtain results after the blood sample is sent to the laboratory. Thus, there is a need for better point of care (POC) testing for blood KB concentration in the newborn. Precision Xtra blood $\beta$ ketone monitoring is such a POC system [3]. It is developed for the measurement of KB for early detection of ketosis in adult and older children with diabetes mellitus [3]. In a study from Japan, Futanani et al. [4] used this device to quantitate blood KB concentrations in the newborn babies between forty-eight and ninety-five hours of life [4]. 
In this study, we examined the ontogeny of blood KB and glucose concentration at 1, 6, 12, 24 and 48 hours of age in full term healthy babies admitted in the normal newborn nursery. To avoid additional prick, babies who were screened for hypoglycemia, metabolic or endocrine disorders, bilirubin measurement or possible sepsis were included. We also investigated if $\mathrm{KB}$ concentrations were affected by the gender, maternal diabetes and the type of feedings. In addition, we studied if $\mathrm{KB}$ concentrations were different in small of gestational age (SGA), large for gestational (LGA), intra-uterine growth restricted (IUGR) when compared to appropriate for gestational age (AGA) and in babies who developed hypoglycemia.

\section{Materials and Methods}

The POCK study was a prospective observational study spanning over a period of one year, April 2018 to March 2019. IRB approval (IHR-2018-MAR-SJ-260) was limited to the babies admitted in the normal newborn nursery. Due to the nature of the study, parental verbal consent was considered adequate. Babies between 35 and 41 weeks of gestation were treated according to our nursery specific protocol. An additional drop of blood was obtained for the measurement of KB and glucose when blood was collected for indications mentioned above. It is the nursery policy to screen all babies who are at risk for developing hypoglycemia at $1,6,12,24$ and 48 hours of age. These include infants of diabetic mothers, (IDM), SGA, LGA and IUGR. Maternal diabetes was treated by the Obstetrician with diet control, oral anti-glycemic drugs, Insulin or a combination of them.

Precision Xtra blood $\beta$-ketone test strips were used for quantitative measure of blood KB $[3,4]$. Instructions provided by the manufacturer were strictly followed which included calibration of the machine using standard ketone solution. Assay range: 0.0 to $8.0 \mathrm{mmol} / \mathrm{L}$, coefficient of variation $3.5 \%$. The strip is calibrated to reflect plasma $\beta-\mathrm{OH}-\mathrm{B}$ using the Randox assay kit (RB1007). Accuracy testing shows that the results compare with the laboratory reference method and are comparable among different users [3]. This Precision KB testing system has been used in a similar study from Japan involving babies between two and four days of age [4]. POC glucose testing was performed by using Accu-Check glucometer (Roche, Inc). Data regarding maternal diabetes, birth weight, gestational age, gender, Apgar score at 1 and 5 minutes, mode of delivery, SGA, LGA or IUGR status and the type of feedings (breast milk or formula) at the time of POC measurements was collected.

Baby with a blood glucose of $<40 \mathrm{mg} / \mathrm{dl}$ was considered hypoglycemic and observed for the signs and symptoms of hypoglycemia. These included abnormal cry, poor feeding, jitteriness or tremors, lethargy, irritability, hypotonia, pallor, cyanosis, tachypnea, apnea or seizures. Irrespective of the symptoms, babies with a blood glucose of $<40 \mathrm{mg} / \mathrm{dl} /$ were treated with additional breast milk or formula feeding. If the glucose concentration did not improve, the baby was transferred to the NICU for further management. Statistical analysis was performed with the help of SPSS (version 20) for Windows package (SPSS Science, Chicago, IL, USA). Description of the data was done in the form of arithmetic mean + /- SD for quantitative data while in the form of frequencies (\%) for qualitative (categorical) data. For quantitative data, unpaired Student's t test was used to test statistical significance of difference between means of variables among two independent groups. For comparison of categorial variables (i.e. to examine the associations between qualitative / quantitative variables) Chisquare test was used if the number of elements in each cell is five or higher. Otherwise Fisher's exact test was used. All data are mean \pm S.D.

\section{Results}

Total of 63 babies (34 M, $29 \mathrm{~F}$ ) were recruited. Gestational ages were $37(n=7), 37-38(n=14), 38-39(n=19)$ and $>39$ (23). There was only one pair of twins. Thirty-eight babies were born by vaginal delivery while 15 and 10 after scheduled or emergency Caesarian section. Twenty-six mothers had a history of diabetes, 37 did not. The average birth weight (BW.) and Apgar scores for all babies were $2961 \pm 500(\mathrm{n}=63)$ and $8(1 \mathrm{~min})$ and $9(5 \mathrm{~min})$. The BW were: AGA $3034 \pm 413(n=51)$, SGA $2287 \pm 213(n=9)$, IUGR $2284 \pm 235(n=5)$ and LGA $3727 \pm 452 n=3$ ). Forty-eight, 48, 46, 45, and 34 babies were exclusively breast fed at 1, 6, 12, 24 and 48 hours of age. There were $0,7,10,10,9$ and 26 babies also receiving formula at 1, 6, 12, 24 and 48 hours of age. There were 9, 5, 3, 3 and o babies receiving formula only at $1,6,12,24$ and 48 hours of age. There were 234 glucose and $\mathrm{KB}$ measurements ( $\sim 3.7$ per baby).

Glucose and KB concentrations at 1, 6, 12, 24 and 48 hours of age are shown in Tables $1 \& 2$. Every blood sample had at least 0.2 mmol/L of KB. At 48 hours of age 15 / 63 ( $24 \%$ ) samples had blood KB > 0.9 (highest, 1.9). In all three groups, AGA, SGA and LGA, the glucose and KB concentrations were highest at 48 hours. Similarly, in IDM and in babies who had developed hypoglycemia, the glucose and KB concentrations were highest at 48 hours of age. In IUGR babies the blood glucose concentration was highest at 48 hours. However, no increase in KB concentration was noted (Table 2). There was no gender difference in KB or glucose concentrations at any age in any groups (data not shown). There were not enough babies receiving only formula. Therefore, we were unable to determine the effect of formula feeding on blood glucose or KB concentration. There was no co-relation between blood glucose and KB levels at any age in any group Nine babies had blood glucose < 40 (range 22-37) within one hour of age. Two babies had persistent hypoglycemia needing transfer to the NICU. One of them was subsequently diagnosed with hyperinsulinism. Only two babies out of 9 with hypoglycemia had signs and symptoms of hypoglycemia which included poor feeding $(n=1)$ and jitteriness $(n=1)$. 
Table 1: Blood glucose and KB concentration in AGA, SGA, LGA and all babies at 1, 6, 12, 24 and 48 hours of age. (Mean \pm SD).

\begin{tabular}{|c|c|c|c|c|c|}
\hline & $1 \mathrm{~h}$ & $6 \mathrm{~h}$ & $12 \mathrm{~h}$ & $24 \mathrm{~h}$ & $48 h$ \\
\hline \multicolumn{6}{|c|}{ AGA $(n=51)$} \\
\hline Glucose & $62 \pm 25$ & $63 \pm 14$ & $69 \pm 15$ & $72 \pm 15$ & $75 \pm 17$ \\
\hline Ketone & $0.54 \pm 0.17$ & $0.49 \pm 0.13$ & $0.47 \pm 0.15$ & $0.56 \pm 0.29$ & $0.81 \pm 0.4$ \\
\hline \multicolumn{6}{|c|}{ SGA (n=9) } \\
\hline Glucose & $59 \pm 16$ & $64 \pm 16$ & $70 \pm 14$ & $69 \pm 16$ & $74 \pm 12$ \\
\hline Ketone & $0,44 \pm 0.15$ & $0.38 \pm 0.04$ & $0.47 \pm 0.1$ & $0.64 \pm 0.31$ & $0.68 \pm 0.32$ \\
\hline \multicolumn{6}{|c|}{ LGA $(n=3)$} \\
\hline Glucose & $58 \pm 30$ & $56 \pm 2$ & $71 \pm 11$ & $76 \pm 6$ & $88 \pm 16$ \\
\hline Ketone & $0.5 \pm 0.17$ & $0.35 \pm 0.07$ & $0.5 \pm 0.1$ & $0.8 \pm 0.1$ & $0.75 \pm 0.35$ \\
\hline \multicolumn{6}{|c|}{ ALL $(n=63)$} \\
\hline Glucose & $61 \pm 24$ & $63 \pm 14$ & $70 \pm 15$ & $72 \pm 14$ & $75 \pm 16$ \\
\hline Ketone & $0.53 \pm 0.17$ & $0.47 \pm 0.13$ & $0.47 \pm 0.14$ & $0.59 \pm 0.3$ & $0.79 \pm 0.39$ \\
\hline
\end{tabular}

Table 2: Blood Glucose and KB concentrations in IUGR, IDM and Hypoglycemic babies at 1, 6, 12, 24 and 48 hours of age. (Mean \pm $\mathrm{SD})$.

\begin{tabular}{|c|c|c|c|c|c|c|}
\hline & $1 \mathbf{h}$ & $\mathbf{6 h}$ & $\mathbf{1 2} \mathbf{~ h}$ & $\mathbf{4 8 ~ h}$ \\
\hline \multicolumn{7}{|c|}{ IUGR (n=5) } \\
\hline Glucose & $58 \pm 13$ & $56 \pm 16$ & $70 \pm 10$ & \multicolumn{2}{|c|}{$72 \pm 7$} & $78 \pm 10$ \\
\hline Ketone & $0.53 \pm 0.11$ & $0,40 \pm 0.01$ & $0.52 \pm 0.1$ & $0.57 \pm 0.15$ & $0.55 \pm 0.17$ \\
\hline \multicolumn{7}{|c|}{ IDM } \\
\hline Glucose & $59 \pm 26(\mathrm{n}=26)$ & $62 \pm 15(\mathrm{n}=23)$ & $68 \pm 11(\mathrm{n}=26)$ & $76 \pm 13(\mathrm{n}=25)$ & $77 \pm 18(\mathrm{n}=25)$ \\
\hline Ketone & $0.57 \pm 0.17(23)$ & $0.50 \pm 0.12(\mathrm{n}=21)$ & $0,48 \pm 0.13(\mathrm{n}=18)$ & $0.53 \pm 0.28(\mathrm{n}=18)$ & $0.81 \pm 0.47(\mathrm{n}=18)$ \\
\hline \multicolumn{7}{|c|}{ HYPOGLYCEMIA } \\
\hline Glucose & $27 \pm 4(\mathrm{n}=9)$ & $58 \pm 5(\mathrm{n}=8)$ & $63 \pm 6(\mathrm{n}=6)$ & $70 \pm 7(\mathrm{n}=6)$ & $77 \pm 6(\mathrm{n}=4)$ \\
\hline Ketone & $0.5 \pm 0.17(\mathrm{n}=9)$ & $0.43 \pm 0.1(\mathrm{n}=8)$ & $0.37 \pm 0.08(\mathrm{n}=6)$ & $0.64 \pm 0.27(\mathrm{n}=6)$ & $0.65 \pm 0.2(\mathrm{n}=4)$ \\
\hline
\end{tabular}

\section{Discussion}

Hypoglycemia is the most common manifestation of failure of metabolic adaptation in the newborn [5]. Hypoglycemia results in a reduced availability of glucose for vital organ function like the heart and may lead to neuro-glycopenia. Many investigators have studied the incidence, clinical manifestations and sequalae of hypoglycemia. Even though there is still controversy regarding the definition of hypoglycemia, glucose of $<40 \mathrm{mg} / \mathrm{dl}$ is considered hypoglycemia for full term babies at our medical center. This number was selected after discussion among the neonatologists and is based on the recent guidelines by the American Academy of Pediatrics.

Precision Xtra blood $\beta$ ketone monitoring is a POC system [3]. When blood is applied to the test strip, $\beta$-OHB reacts with the chemicals on the test strip producing a small electric current which is measured. And the result is displayed on the meter after ten second. The size of the current depends on the amount of $\beta$-OHB. It is practical and reliable. Therefore, similar to Futatani, we chose to use this POC device.

Melichar [6] observed average serum KB levels of 0.16 and $1.0 \mathrm{mmol} / \mathrm{L}$ at 1-12 hours and 1-7 days of life respectively, while
Personn et al. [7] reported average levels of 0.49 (5-24 hours), 0.82 (1-2 days) and 0.9 (2-3 days) mmol/L [7]. Hawdon et al. [8] studied ontogeny of serum KB concentration during the first six days of life among full term AGA neonates [8]. The average concentrations were 0.04 (1-12 hours), 0.21 (12-24 hours), 0.41 (day 2) and 0.43 (day 3). Futatani [4] using Precision Xtra ketone POC system, measured KB levels in the healthy newborns between 48 and 95 hours of life who were exclusively breast fed [4]. The levels ranged from $\sim 0.05$ to $5.5 \mathrm{mmol} / \mathrm{L}$ We quantitated $\mathrm{KB}$ concentrations at 1 , $6,12,24$ and 48 hours of life in AGA, SGA, LGA, IUGR and IDM who also received supplemental formula. Blood KB levels ranged from 0.2 to $1.9 \mathrm{mmol} / \mathrm{L}$. KB concentrations among all these studies seem to be similar. Nonetheless, some of the apparent variations may be due to differences in the ethnicity of the newborn population, the maternal food intake and habits, neonatal feeding practices, short half-life ( 1.6 hours) of KB, methods used to measure $\mathrm{KB}$ or a combination of these.

In studies by Melchar, Personn and Hawdon serum KB concentrations increased with age during the immediate newborn period. While KB concentrations in our study were highest at 48 hours in all groups except IUGR, the apparent increase was not 
statistically significant as compared to earlier ages. Similar to our observations, Futatani did not find a co-relation between KB concentration and postnatal age [4]. Warshaw [9] examined serum $\mathrm{KB}$ concentration in breast fed and formula fed term newborn infants [9]. They concluded that breast fed babies had higher levels of KB compared to formula fed infants. Hawdon et al. [8] also observed that breast fed infants had higher levels of KB concentrations [8]. Futatani reported blood KB concentrations in neonates who were breast fed [4]. They did not study the effect of exclusive or supplemental formula feedings. In our study we could not determine the effect of formula feeding on the blood KB concentrations since very few babies received exclusively formula feedings.

Bougneres [10] studied KB transport in the human neonates and infants [10]. They concluded KB play an important role in the CNS metabolism of the term newborn baby [10]. Hawdon [11] also studied ontogeny of serum blood glucose concentration during the first six days of life among full term AGA babies [11] The overall incidence of hypoglycemia (blood glucose $<40 \mathrm{mg} / \mathrm{dl}$ ) was $12 \%$ (comparable to our incidence of $14 \%$. Some neonates had low blood glucose and high KB concentration [11]. There was a significant negative relationship between $\mathrm{KB}$ and glucose concentrations for infants 2-3 days old. However, there was no such relationship for 1 day or those older than 3 days. Some infants with hypoglycemia were unable to mount a ketogenic response. The ability of the neonatal CNS to utilize KB is neuroprotective during episodes of hypoglycemia. In that study infant's plasma KB increase to a maximum on 2-3 days of life. In 48 to 95 hours old exclusively breast-fed neonates, Futatani found a strong negative co-relation between blood glucose and KB concentration [4]. They proposed that breastfed infants can tolerate lower glucose because the cerebral uptake of KB is related to the blood concentration. In our study, $14 \%$ of all babies became hypoglycemic within the first hour of life. There was no increase in KB concentration or any co-relation between blood KB and glucose levels. Our findings are similar to those by Hawdon [11].

Hawdon [11] studied hormonal and metabolic response to hypoglycemia in SGA infants [11]. Plasma KB levels were low in SGA infants. The negative relationship between blood glucose and KB [11], was absent. SGA infants had low lipolytic and ketogenic capacity. Hawdon concluded that all asymptomatic term infants with blood glucose concentration less than $<40 \mathrm{mg} / \mathrm{dl}$ need not be treated with IV glucose as there is evidence that when the infants are healthy and AGA, KB production provides alternative fuels for brain [11]. However, infants who are sick, SGA or have persistent or symptomatic hypoglycemia should be investigated and treated. In our study IUGR but not SGA infants had low KB concentration especially at 48 hours of age.

In summary, we have established the ontogeny of blood $\mathrm{KB}$ levels using POC device within the first 48 hours of life. Future studies should be undertaken to establish blood $\mathrm{KB}$ levels in premature babies with and without respiratory failure and in those treated with lipid emulsions as part of the total parental nutrition.

\section{Acknowledgement}

There was no conflict of interest. The funding was provided by the division of Neonatology at UCLA. We thank all the parents for allowing their neonates to be enrolled in this study. We are truly indebted to the entire nursing staff for their help.

\section{References}

1. de Rooy La, Hawdon J (2003) Nutritional factors that affect the postnatal metabolic adaptation of full term small and large for gestational infants. Pediatrics 109(3): 1-8.

2. Ward Platt M, Deshpande S (2005) Metabolic adaptation at birth Seminars in Fetal and Neonatal Medicine 10(4): 341-350.

3. (2012) Precision Xtra blood $\beta$ ketone test strips monitoring. Abbott Bulletin pp. 1-4

4. Futatani T, Shimao A, Ina S, Higashiyama H, Fujita S, et al. (2017) Capillary blood ketone levels as an indicator of inadequate breast milk intake in the early neonatal period. J of Pediatr 191: 76-81.

5. Rozance PJ, Hay WW (2006) Hypoglycemia in newborn infants: Features associated with adverse outcomes. Biol Neonate 90(2): 74-86.

6. Melichar V, Drahota Z, Hahn P (1965) Changes in the blood levels of acetoacetate and ketone bodies in newborn infants. Biol. Neonat 8: 348352.

7. Personn B, Gentz J (1966) The pattern of blood lipids, glycerol and KB during the neonatal period. Infancy and childhood. Acta Pediatrica Scandinavica 55(4): 353-362.

8. Hawdon JM, Ward Platt MP, Aynsley Green A (1992) Patterns of metabolic adaptation for preterm and term infants in the first neonatal week. Archives of Diseases of Childhood 67: 357-365.

9. Warshaw JW, Curry E (1980) Comparison of serum carnitine and ketone body concentrations in breast and in formula fed newborn infants. J Pediatr 97(1): 122-225.

10. Bougneres PF, Lemmel C, Ferre P, Bier, DM (1986) Ketone body transport in the human neonate and infant. J Clin Invest 77(1): 42-48. 
ISSN: 2574-1241

DOI: 10.26717/BJSTR.2021.36.005871

Uday P Devaskar. Biomed J Sci \& Tech Res

(c) (P) This work is licensed under Creative

Submission Link: https://biomedres.us/submit-manuscript.php

$\begin{array}{ll}\text { BIOMEDICAL } & \text { Assets of Publishing with us } \\ \text { RESEARCHES } & \text { - Global archiving of articles } \\ & \text { - Immediate, unrestricted online access } \\ & \text { - Rigorous Peer Review Process } \\ & \text { - Authors Retain Copyrights }\end{array}$

